\section{Eficácia da associação de cipermetrina, clorpirifós, butóxido de piperonila e fluazuron contra haematobia irritans em bovinos naturalmente infestados}

\author{
Effecacy of the association of cipermetrin, chlorpyriphos, \\ piperonyl butoxide and fluazuron against haematobia irritans \\ in naturally infested cattle
}

\begin{abstract}
Diefrey Ribeiro Campos', Barbara Rauta de Avelar', Gabriela Ferreira de Oliveira', Mariana Silva Revoredo Alves², Raphael Comissário Melo ${ }^{2}$, Monique Taveira Medeiros², Katherina Comendouros ${ }^{3} \&$ Fabio Barbour Scott ${ }^{3 *}$

'Médicos veterinários, MSc. Programa de Pós-graduação em Ciências Veterinárias, Universidade Federal Rural do Rio de Janeiro - UFRRJ, Seropédica, RJ, Brasil

2Médicos veterinários. Programa de Pós-graduação em Ciências Veterinárias, Universidade Federal Rural do Rio de Janeiro UFRRJ, Seropédica, RJ, Brasil

${ }^{3}$ Médicos veterinários, DSc, Professores. Departamento de Parasitologia Animal do Instituto de Veterinária, Universidade Federal Rural do Rio de Janeiro - UFRRJ, Seropédica, RJ, Brasil
\end{abstract}

\section{Resumo}

Haematobia irritans é uma mosca de grande importância na pecuária bovina. É responsável por causar estresse crônico ao rebanho causando desconforto e levando a redução da produção de carne e leite e depreciação do couro. Também são responsáveis por transmitir vírus, bactéria e facilitar ao desenvolvimento de míiases. O objetivo deste trabalho foi avaliar a eficácia de um produto ectoparasiticida contendo a associação de cipermetrina, clorpirifós, butóxido de pireronila e fluazuron na formulação "pour on" no controle da mosca $H$. irritans em bovinos naturalmente infestados. Foram utilizados 30 bovinos, mestiços, sem distinção de sexo ou idade. Todos os animais incluídos no estudo apresentaram pelo menos 50 moscas adultas de $H$. irritans de ocorrência natural. Os animais do grupo tratado foram medicados com a associação de cipermetrina ( $5 \mathrm{mg} / \mathrm{kg}$ ), clorpirifós (7 mg/kg), butóxido de pireronila ( $5 \mathrm{mg} / \mathrm{kg}$ ) e fluazuron (2,5 mg/kg), na dose de $1 \mathrm{~mL}$ para cada 10 quilos de peso vivo, pela via tópica ("pour on") em dose única. A eficácia mosquicida foi avaliada um, três, sete, 14 e 21 dias após o tratamento, a partir da contagem de moscas adultas sobre os animais dos dois os grupos. A eficácia da associação testada neste estudo foi de 98,65\%; 96,69\%; 92,01\%; 87,79\% e 76,43\% nos dias +1, +3, +7, +14 e +21 respectivamente. A associação de cipermetrina, clorpirifós, butóxido de piperonila e fluazuron na formulação "pour on" é eficaz no controle de $H$. irritans em bovinos naturalmente infestados.

Palavras-chave: controle, mosca do chifre, piretróide, organofosforado.

\begin{abstract}
Haematobia irritans is a fly of great importance in cattle breeding. It is responsible for causing chronic stress due to discomfort and leading to reduced meat and milk production and depreciation of leather. They are also responsible for transmitting viruses, bacteria and facilitating the development of myiasis. The objective of this study was to evaluate the efficacy of a product ectoparasiticide containing a association of cypermethrin, chlorpyrifos, butoxide pireronila and fluazuron in formulating "pour on" the fly control $H$. irritans in cattle naturally infested. Were used thirty crossbred cattle, regardless of sex or age. All animals included in the study had at least 50 naturally occurring adult $H$. irritans flies. The animals in the treated group were medicated with the combination of cypermethrin $(5 \mathrm{mg} / \mathrm{kg})$, chlorpyrifos $(7 \mathrm{mg} / \mathrm{kg})$, pireronyl butoxide ( $5 \mathrm{mg} / \mathrm{kg})$ and fluazuron $(2.5 \mathrm{mg} / \mathrm{kg})$ at a dose of $1 \mathrm{~mL}$ for every $10 \mathrm{~kg}$ live weight, "pour on" in single-dose. Efficacy was evaluated one, three, seven, 14 and 21 days after treatment, from the adult flies count on the animals of the two groups. The efficacy of the association tested in this study was 98.65\%; $96.69 \%$; $92.01 \% ; 87.79 \%$ and $76.43 \%$ on days $+1,+3,+7,+14$ and +21 respectively. The association of cypermethrin, chlorpyrifos, pyreronyl butoxide and fluazuron in the pour-on formulation is effective in controlling $H$. irritans in naturally infested cattle.
\end{abstract}

Keywords: control, horn fly, pyrethroid, organosphosphorous.

\section{BJ $\mathrm{M}$ \\ Brazilian Journal of Veterinary Medicine}

p-ISSN 0100-2430

e-ISSN 2527-2179

อ
Como citar: Campos, D. R., Avelar, B. R., Oliveira, G. F., Alves, M. S. R., Melo, R. C., Medeiros, M. T., Comendouros, K., \&Scott, F. B. (2017). Eficácia da associação de cipermetrina, clorpirifós, butóxido de piperonila e fluazuron contra haematobia irritans em bovinos naturalmente infestados. Brazilian Journal of Veterinary Medicine, 39(3), 160-164. doi: 10.29374/2527-2179.bjvm014517

Fonte de financiamento: Fundação de Apoio à Pesquisa Científica e Tecnológica da Universidade Federal Rural do Rio de Janeiro.

Conflito de interesses: Os autores declaram não haver conflito de interesses que precisam ser informados

Recebido: Março 22, 2017.

Aceito: Setembro 30, 2017

O estudo foi realizado na Universidade Federal Rural do Rio de Janeiro - UFRRJ, Seropédica, RJ, Brasil.

\section{${ }^{*}$ Correspondência}

Fabio Barbour Scott

Programa de Pós-graduação em Ciências Veterinárias, Instituto de Veterinária, Universidade Federal Rural do Rio de Janeiro - UFRRJ

Rod. BR 465, Km 7

CEP 23897-035 - Seropédica (RJ), Brasil

E-mail:scott.fabio@gmail.com

Copyright Campos et al. Este é um artigo publicado em acesso aberto (Open Access) sob a licença Creative Commons Attribution Non-Commercial, que permite uso, distribuição e reprodução em qualque meio, sem restrições desde que sem fins comerciais e que o trabalho original seja corretamente citado. 


\section{Introdução}

Haematobia irritans (Diptera:Muscidae) éuma mosca hematófaga conhecida popularmente no Brasil como mosca-do-chifre. Foi introduzida no Brasil na década de 70, e se adaptou muito bem as condições climáticas e a criação extensiva de gado do país. Estando, portanto, amplamente distribuída pelo território nacional desde a década de 80 (Campbell \& Thomas, 1992; Brito et al., 2005).

Essa mosca causa grande prejuízo econômico a pecuária bovina. A perda de sangue pela a hematofagia não é o maior problema causado ao rebanho, e sim a irritabilidade provocada pela picada dessa mosca leva ao quadro de estresse crônico. Os animais passam mais tempo tentando se livrar dos insetos, do que se alimentando, resultando em menor conversão alimentar, perda de peso, queda na produção de carne e leite, acarretando em um prejuízo de \$2,56 bilhões (Grisi et al., 2002; Grisi et al., 2014). Outra perda ocasionada por este ectoparasito está relacionada a menor qualidade do couto. A picada dessa mosca provoca uma reação inflamatória local que torna a pele espessa e reduz sua flexibilidade (Guglielmone et al., 1999; Cupp et al., 2000; Maciel et al., 2015).

Além dos problemas descritos acima, H. irritans também é responsável por transmitir o carbúnculo hemático, leucose bovina e anaplasmose (Foil \& Gorham, 2000; Hornok et al., 2008). As feridas que estas moscas causam na pele predispõe ao desenvolvimento de míiases por Cochliomyia hominivorax e também são considerados vetores foréticos de Dermatobia hominis (Brito et al., 2005; Bianchin \& Alves, 2002).

O controle químico desta mosca foi realizado principalmente com organoclorados, organofosforados e piretróides nas décadas de 40, 50, 70 e 80. Com o descobrimento das lactonas macrocíclicas essas também passaram a ser utilizada no controle da mosca do chifre (Guglielmone et al., 2001; Andrade et al., 2012).

Atualmentejá existem relatos de H. irritans resistentes a estes quatros princípios ativos citados anteriormente. Portanto a busca de novas moléculas ou associações são necessárias para o controle desta mosca (Sparks et al., 1985; Oyarzún et al., 2008; Stafford \& Coles, 2017).

A cipermetrina é um piretróide sintético derivado de plantas do gênero Chrysanthemum com excelente potencial acaricida e inseticida, além de possuir ação repelente. Seu mecanismo de ação está ligado ao bloqueio dos canais de sódio e causar hiperexcitação de insetos. O clopirifós é um organofosforado que também possui ação acaricida e inseticida, seu mecanismo de ação está ligado ao bloqueio da enzima acetilcolinesterase levando o parasito à morte por paralisia (Taylor, 2001).

O butóxido de piperonila (PBO) é amplamente utilizado na agricultura para o controle de pragas agrícolas que apresentam resistência aos organofosforados e piretróides. Este reduz a metabolização destas drogas pelo parasito, aumentando assim a suscetibilidade do mesmo (Beckel et al., 2006).

O fluazuron é um regulador de crescimento de artrópodes que age inibindo a síntese de quitina. Vem sido utilizado na pecuária para o controle do carrapato Rhipicephalus microplus (Bull et al., 1996).

O objetivo deste trabalho foi avaliar a eficácia de um produto ectoparasiticida contendo a associação de cipermetrina, clorpirifós, PBO e fluazuron na formulação "pour on" no controle da mosca Haematobia irritans em bovinos naturalmente infestados.

\section{Materiais e métodos}

Este estudo foi aprovado pelo Comitê de Ética de Uso de Animal (CEUA) da Fundação de Apoio à Pesquisa Científica e Tecnológica (FAPUR), da UFRRJ, realizada no dia 21 de agosto de 2013.

Para este estudo foram utilizados 30 bovinos, mestiços das raças Gir e Holandês, machos e fêmeas, com peso variando de 253 a 304 quilos e identificados por brincos numerados nas duas orelhas.

O ranqueamento do estudo foi realizado com base na contagem de moscas em cada animal. Os dois animais que apresentaram maior número de moscas foram sorteados um para cada grupo e o procedimento foi repetido até que estivesse completa as 15 repetições, compondo dois grupos: controle e tratado. 
No dia do tratamento, os bovinos foram pesados e os animais do grupo tratado foram medicados com a associação de cipermetrina ( $5 \mathrm{mg} / \mathrm{kg}$ ), clorpirifós ( $7 \mathrm{mg} / \mathrm{kg}$ ), butóxido de pireronila $(5 \mathrm{mg} / \mathrm{kg}$ ) efluazuron (2,5 mg $/ \mathrm{kg}$ ), na dose de $1 \mathrm{~mL}$ para cada $10 \mathrm{Kg}$ de peso corporal, pela via tópica ("pour on") em dose única (Couro Limpo ${ }^{\circledR}$ - Noxon Do Brasil Química e Farmacêutica Ltda). Os animais do grupo controle não receberam nenhum tipo de tratamento.

A avaliação da eficácia foi realizada com um, três, sete, 14 e 21 dias após o tratamento, a partir da contagem de moscas vivas presentes nos animais do grupo controle e tratado. O cálculo da eficácia foi realizado com base na fórmula abaixo:

Eficácia (\%) = 100x [(média aritmética de moscas do grupo controle - média aritmética de moscas do grupo tratado) / (média aritmética de moscas do grupo controle)].

A análise estatística foi realizada pelo programa estatístico computacional BioStat 5.0, através de comparação das médias. Inicialmente foi realizada avaliação quanto a distribuição dos dados. Após verificação que eram dados não paramétricos optou-se pelo método de Mann Whitney. O nível de significância considerado foi de $\mathrm{p} \leq 0,05$ (95\%).

\section{Resultados}

A média da contagem de moscas vivas antes do tratamento no grupo controle foi de 79,07 $\pm 23,48$, enquanto nos animais do grupo tratado foi $78,27 \pm 19,66$. Não sendo verificada diferença estatística ( $>0,05)$ sobre as médicas dos dois grupos.

O número médio de moscas vivas no grupo controle foi de: 69,13 para o $\mathrm{D}+1 ; 70,60$ para o $\mathrm{D}+3$, 71,73 para o $\mathrm{D}+7 ; 66,07$ para o $\mathrm{D}+14 ; 68,73$ para o $\mathrm{D}+21$. Para o grupo tratado o número médio de moscas vivas foi de: 0,$93 ; 2,33 ; 5,73 ; 8,07 ; 16,20$ para os dias um, três, sete, 14 e 21 após o tratamento. Foi observado diferença estatística $(\mathrm{p}<0,05)$ em todos os dias experimentais após o tratamento.

A formulação apresentou eficácia mosquicida de 98,65\%; 96,69\%; 92,01\%; 87,79\% e 76,43\% nos dias $+1,+3,+7,+14$ e +21 respectivamente e os resultados estão demonstrados na Tabela 1 .

\section{Discussão e conclusão}

A mosca do chifre é um ectoparasito que ainda traz grandes prejuízos a pecuária brasileira (Grisi et al., 2014) e a sua principal forma de controle ainda é o uso de ectoparasiticidas (Oyarzún et al., 2008).

A formulação testada neste estudo apresentou eficácia no controle de H. irritans por até14 dias após o tratamento, e seu declínio iniciou a partir do dia +21 . Apesar dos constantes registros de resistência da mosca do chifre a piretróides e organofosforados, a formulação estudada manteve eficácia frente a este parasito. Provavelmente a queda da eficácia está ligada a perda do efeito residual do produto, fazendo que o número de moscas aumentasse no grupo tratado.

Não existem relatos da resistência cruzada entre organofosforados e piretróides (Brito et al., 2005) para H. irritans. A associação dos dois em uma única formulação permite a eliminação

Tabela 1. Média aritmética das contagens de moscas do chifre (Haematobia irritans) vivas nos bovinos do grupo controle e medicado antes do tratamento e um, três, sete, 14 e 21 dias após o tratamento com a associação de cipermetrina, clorpirifós, butóxido de pireronila e fluazuron na formulação "pour on".

\begin{tabular}{|c|c|c|c|c|}
\hline \multirow{2}{*}{ Dia Experimental } & \multirow{2}{*}{$\begin{array}{c}\text { Grupo Controle } \\
\text { Média* }\end{array}$} & \multicolumn{2}{|c|}{ Grupo Tratado } & \multirow{2}{*}{$p$-value } \\
\hline & & Média* & Eficácia (\%) & \\
\hline 0 & $79,07 a \pm 23,48$ & $78,27 \mathrm{a} \pm 16,66$ & - & \\
\hline 1 & $69,13^{\mathrm{a}} \pm 25,13$ & $0,93^{\mathrm{b}} \pm 0,93$ & 98,65 & 0,0001 \\
\hline 3 & $70,60^{a} \pm 27,62$ & $2,33^{b} \pm 1,58$ & 96,69 & 0,0001 \\
\hline 7 & $71,73 \pm 22,49$ & $5,73^{b} \pm 3,59$ & 92,01 & 0,0001 \\
\hline 14 & $66,07^{a} \pm 22,89$ & $8,07^{b} \pm 6,65$ & 87,79 & 0,0001 \\
\hline 21 & $68,73^{\mathrm{a}} \pm 23,91$ & $16,20^{\mathrm{b}} \pm 10,04$ & 76,43 & 0,0001 \\
\hline
\end{tabular}

*Média Aritmética; Letras diferentes ab significa diferença estatística $(p<0,05)$ entre as médias. 
de moscas que são resistentes aos piretróides. Além disso, a associação com o PBO aumenta a sensibilidade de insetos a estes dois princípios ativos, sendo indicado sua associação na suspeita de populações de insetos resistentes (Beckel et al., 2006).

O fluazuron éum regulador de crescimento de artrópodes e tem como função inibir a síntese de quitina (Bull et al., 1996; Taylor, 2001). Este tipo de ectoparasiticida apresenta pouca ação adulticida, porém seu uso é recomendado para controle das formas não parasitárias presentes no ambiente. A ação do fluazuron não foi avaliada neste estudo. Este fármaco veem sendo empregado de forma isolada ou em associação com moléculas que tenham características inseticidas/acaricidas.

Outros reguladores de crescimento de artrópodes, como diflubenzuron, metoprene e fenoxicarb já foram avaliados para o controle de H. irritans. Estes interferem no ciclo da mosca formando ovos, larvas e pupas anômalos impedindo que se tornem adultos (Silva \& Mendes, 2002; Silva et al., 2004).

A associação de um piretróide com um organofosforado mais um agente sinérgico e um IGR como no caso do presente, quando empregado em situações manejo para o controle de diversos ectoparasitos de bovinos, poderá ser uma ferramenta importante para o controle sanitário do rebanho. Destaca-se que para o controle efetivo das parasitoses que acometem um rebanho de bovinos é de suma importância que todas as medidas necessárias sejam tomadas, tendo em vista que não basta somente a aplicação de uma formulação ectoparasiticida efetiva. Ações como limpeza das pastagens, conhecimentos epidemiológicos dos parasitos que acometem o rebanho, a compreensão da susceptibilidade da população a ser combatida, a escolha correta da formulação a ser empregada assim como do momento mais adequado dentre outros, constituem-se em premissas essências para o sucesso no controle das parasitoses dos bovinos.

Com base nos dados obtidos neste estudo, pode-se concluir que a associação de cipermetrina, clorpirifós, PBO e fluazuron na formulação "pour on" é eficaz no controle de H. irritans em bovinos naturalmente infestados.

\section{Referências}

Andrade, B. M. A., Rosa, P. E. J., Soares, V. E., Souza, L. M., Mota, F. C. D., Giamlorenço, T. F., \& Gírio, T. M. S. (2012), Eficácia de diferentes formulações no controle da mosca Haematobia irritans em bovinos naturalmente infestados. Bioscience Journal, 28, 354-359.

Beckel, H., Lorini, I., \& Lazzari, S. M. (2006). Efeito do sinergista butóxido de piperonila na resistência de Oryzaephilus surinamensis (L.)(Coleoptera, Silvanidae) a deltametrina e fenitrotiom. Revista Brasileira de Entomologia, 50(1), 110-114. http://dx.doi.org/10.1590/S0085-56262006000100016.

Bianchin, I., \& Alves, R. G. (2002). Mosca-dos-chifres, Haematobia irritans: comportamento e danos em vacas e bezerros Nelore antes da desmama. Pesquisa Veterinária Brasileira, 22(3), 109-113. http://dx.doi.org/10.1590/ s0100-736X2002000300004.

Brito, L. G. B., Borja, G. E. M., Oliveira, M. D. S., \& Netto, F. S. (2005). Mosca-dos-chifres: aspectos bio-ecológicos, importância econômica, interações parasito-hospedeiro e controle. Rondônia: Embrapa.

Bull, M. S., Swindale, S., Overend, D., \& Hess, E. A. (1996). Suppression of Boophilus microplus populations with fluazuron-an acarine growth regulator. Australian Veterinary Journal, 74(6), 468-470. http://dx.doi. org/10.1111/j.1751-0813.1996.tb07575.x. PMid:9006870.

Campbell, J. B., \& Thomas, G. D. (1992). The history, biology, economics, and control of the Horn Fly, Haematobia irritans. Agri-practice, 13, 31-36.

Cupp, M. S., Zhang, D., \& Cupp, E. W. (2000). Horn fly (Diptera: Muscidae) saliva targets thrombin action in hemostasis. Journal of Medical Entomology, 37(3), 416-421. http://dx.doi.org/10.1093/imedent/37.3.416. PMid:15535586.

Foil, L. D., \& Gorham, J. R. (2000). Mechanical transmission of disease agents by arthropods. In B. F. Eldridge \& J. D. Edman (Eds.), Medical entomology. Dordrecht: Springer.

Grisi, L., Leite, R. C., Martins, J. R., Barros, A. T., Andreotti, R., Cançado, P. H., León, A. A., Pereira, J. B., \& Villela, H. S. (2014). Reassessment of the potential economic impact of cattle parasites in Brazil. Revista Brasileira de Parasitologia Veterinária, 23(2), 150-156. http://dx.doi.org/10.1590/S1984-29612014042. PMid:25054492.

Grisi, L., Massard, C. L., Moya-Borja, G. E., \& Pereira, J. B. (2002). Impacto econômico das principais ectoparasitoses em bovinos no Brasil. A Hora Veterinária, 21, 8-10.

Guglielmone, A. A., Castelli, M. E., Volpogni, M. M., Medus, P. D., Martins, J. R., Suárez, V. H., Anziani, O. S., \& Mangold, A. J. (2001). Toxicity of cypermethrin and diazinon to Haematobia irritans (Diptera: Muscidae) in its American southern range. Veterinary Parasitology, 101(1), 67-73. http://dx.doi.org/10.1016/S03044017(01)00490-3. PMid:11587834. 
Guglielmone, A. A., Gimeno, E., Idiart, J., Fisher, W. F., Volpogni, M. M., Quaino, O., Anziani, O. S., Flores, S. G., \& Warnke, O. (1999). Skin lesions and cattle hide damage from Haematobia irritans infestations. Medical and Veterinary Entomology, 13(3), 324-329. http://dx.doi.org/10.1046/i.1365-2915.1999.00167.x. PMid:10514060.

Hornok, S., Földvári, G., Elek, V., Naranjo, V., Farkas, R., \& de la Fuente, J. (2008). Molecular identification of Anaplasma marginale and rickettsial endosymbionts in blood-sucking flies (Diptera: Tabanidae, Muscidae) and hard ticks (Acari: Ixodidae). Veterinary Parasitology, 154(3-4), 354-359. http://dx.doi.org/10.1016/j. vetpar.2008.03.019. PMid:18495345.

Maciel, W., Lopes, W. D., Cruz, B., Teixeira, W., Felippelli, G., Sakamoto, C. A., Fávero, F. C., Buzzulini, C., Soares, V., Gomes, L. V., Bichuette, M., \& Costa, A. J. (2015). Effects of Haematobia irritans infestation on weight gain of Nelore calves assessed with different antiparasitic treatment schemes. Preventive Veterinary Medicine, 118(1), 182-186. http://dx.doi.org/10.1016/j.prevetmed.2014.11.006. PMid:25465474.

Oyarzún, M. P., Quiroz, A., \& Birkett, M. A. (2008). Insecticide resistance in the horn fly: alternative control strategies. Medical and Veterinary Entomology, 22(3), 188-202. http://dx.doi.org/10.1111/j.1365-2915.2008.00733.x. PMid:18816268.

Silva, J. J., \& Mendes, J. (2002). Effect of diflubenzuron on immature stages of Haematobia irritans (L.) (Diptera: Muscidae) in Uberlandia, State of Minas Gerais, Brazil. Memórias do Instituto Oswaldo Cruz, 97(5), 679-682. http://dx.doi.org/10.1590/S0074-02762002000500017. PMid:12219135.

Silva, J. J., Mendes, J., \& Lomônaco, C. (2004). Developmental stress by Diflubenzuron in Haematobia irritans (L.) (Diptera: Muscidae).Neotropical Entomology,33(2), 249-253. http://dx.doi.org/10.1590/S1519-566X2004000200017.

Sparks, T. C., Quisenberry, S. S., Lockwood, J. A., Byford, R. L., \& Roush, R. T. (1985). Insecticide resistance in the horn fly, Haematobia irritans. Journal of Agricultural Entomology, 2, 217-233.

Stafford, K. A., \& Coles, G. C. C. (2017). Drug resistance in ectoparasites of medical and veterinary importance. Switzerland: Springer. http://dx.doi.org/10.1007/978-3-319-46718-4_48.

Taylor, M. A. (2001). Recent developments in ectoparasiticides. Veterinary Journal, 161(3), 253-268. http://dx.doi. org/10.1053/tvjl.2000.0549. PMid:11352483. 